\title{
Upper Alpha Neurofeedback Training Over the Motor Cortex Increases SMR Desynchronization in Motor Tasks
}

\author{
Eduardo López-Larraz, Carlos Escolano, Javier Minguez
}

\begin{abstract}
Desynchronization of sensorimotor rhythms (SMR) is a distinctive feature that provides a discriminative pattern for BCI operation. However, individuals such as BCI illiterates can not produce these discriminable patterns with sufficient reliability. Additionally, SMR desynchronization can become deteriorated or extinct in patients with spinal cord injury or a cerebrovascular accident. In all these situations BCI usage is compromised. This paper proposes an intervention based on neurofeedback training of the upper alpha band to improve SMR desynchronization. The feasibility of this intervention is demonstrated in a preliminary study in which five healthy subjects were trained to increase their upper alpha band power. Such increases produced higher SMR desynchronization and better discrimination between rest and execution states of a motor task.
\end{abstract}

\section{INTRODUCTION}

Execution of motor tasks with a given limb generates a desynchronization (i.e., a reduction in the average power) of sensorimotor-rhythms (SMR) over the somatotopic motor cortex. This phenomenon can be measured in the electroencephalographic activity (EEG) [1]. This pattern of motor brain activity can be also elicited either by motor attempt or by motor imagery [2] (both with different neural circuits and partially different EEG activity). Brain-computer interfaces (BCIs) have recently emerged as a technology to translate user intentions into commands with applications in many fields, such as in neuro-robotics or neuro-prosthetics [3]. Many of the BCIs developed to date are based on the decoding of motor intentions using the activity of the motor cortex. Thus, the application of BCI technology requires the user to be able to produce certain recognizable motor brain patterns (i.e., SMR desynchronization generated by motor attempt or motor imagery). However, this is not always the case in both healthy subjects and patients.

On one hand, a large portion of healthy subjects (approximately $20 \%$ of population) is not able to use motor imagery BCI technology (referred to as BCI illiterates) because they do not produce reliable and stable EEG patterns, and thus the process can not be accurately detected [4]. On the other hand, a large portion of BCIs are being developed for spinal cord injury (SCI) and cerebrovascular accident (CVA) patients. In the case of SCI, several research studies have explored the changes produced in the brain of SCI patients after years of chronic injury. Some studies found

E. López-Larraz, C. Escolano, and J. Minguez are with the Instituto de Investigación en Ingeniería de Aragón (I3A) and Dpto. de Informática e Ingeniería de Sistemas (DIIS), Universidad de Zaragoza, Spain. J. Minguez is also with Bit\&Brain Technologies SL, Spain. E-mail: \{edulop, cescolan, jminguez\}@unizar.es. This work has been partially supported by projects HYPER-CSD2009-00067 and DPI2009-14732-C02-01 of the Spanish Government, and by DGA-FSE (grupo T04) that SCI patients produced weak (or none) detectable SMR activity on the motor cortex during an attempt of moving their paralyzed limbs, specially in the chronic state [2], [5]. In the case of CVA, the brain neural networks are damaged. This damage could hinder the detection of motor-related brain activity in injured areas and their vicinity (contralateral muscle activity). It has been proved that during the execution of movement both the contralateral and ipsilateral cortices are active [6], and the use of the healthy hemisphere has been recently proposed to decode motor intention in patients who suffered a stroke [7]. While the results are promising, the decoding results are attenuated by the fact that the activation of the ipsilateral cortex is always less significant than the contralateral. In the three mentioned cases (healthy BCI illiterates, SCI and CVA patients), a possible solution could be to develop an intervention to improve or reconstruct the motor-related EEG activity prior to BCI usage [8]. In this direction, this paper proposes a preliminary study with five healthy subjects to build a possible intervention based on neurofeedback training.

Neurofeedback (NF) training is a biofeedback modality that allows the user to change specific brain rhythms by means of an operant conditioning paradigm. In this study a NF training was designed to increase upper alpha (UA) power, and its different effects were explored: $(i)$ The tendencies in the trained EEG variable (i.e., UA band) during the NF procedure. Some NF studies demonstrated trainability of UA over parieto-occipital areas of the scalp in contexts of cognitive enhancement [9], [10]. (ii) The changes in EEG activity in the execution of an active mental task, and during the execution of a motor task. Most NF studies related to motor contexts focused on decreasing SMR power (i.e., augmenting desynchronization) during the motor tasks [11], [12]. This paper, however, focuses on increasing UA, following studies [10], [13], which reported increases in desynchronization during the execution of cognitive tasks after NF training for UA enhancement. (iii) The implications that the EEG changes may have for BCI operation. [4] stated that subjects with higher alpha power in basal states could lead to better performances in SMR based BCIs. (iv) The improved motor performance that may be achieved by the higher desynchronization ability. Recent evidence revealed that subjects presented shorter reaction times during a motor task while voluntarily decreasing SMR patterns [14].

\section{Methods}

\section{A. Participants and Experimental Design}

Five healthy subjects (four males and one female) participated in the study. They were duly informed about the 


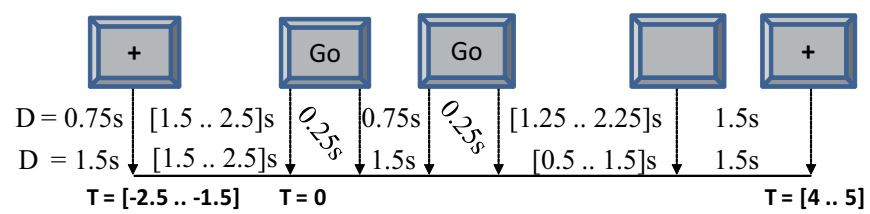

Fig. 1. Design of the Go/No-Go task. D indicates the two different time intervals that randomly separate the warning and imperative stimuli.

entire protocol and gave informed consent. The experiment consisted of five NF training sessions executed in five consecutive days, one session per day. Participants executed, for each session, five NF training trials of five minutes each. EEG screenings were performed immediately before and after each training session to assess changes in the EEG. In addition, a motor assessment (sec. II-D) was carried out at the beginning of the first training session, and at the end of the last training session, to assess changes both in motor behavior and in EEG patterns due to NF application.

\section{B. Signal Acquisition}

EEG signals were recorded from 16 active electrodes placed at FP1, FP2, F3, Fz, F4, C3, Cz, C4, CP3, CPz, $\mathrm{CP} 4, \mathrm{P} 3, \mathrm{Pz}, \mathrm{P} 4, \mathrm{O} 1$ and $\mathrm{O} 2$ (according to the international $10 / 10$ system). Ground and reference electrodes were placed on FPz and on the left earlobe, respectively. EEG was amplified using a commercial gTec system at a sampling frequency of $256 \mathrm{~Hz}$, power-line notch-filtered at $50 \mathrm{~Hz}$, and bandpass-filtered between 0.5 and $60 \mathrm{~Hz}$. Signal acquisition, processing and feedback presentation were developed using Bit\&Brain Technologies software.

\section{Neurofeedback Procedure}

Screening: Screening consisted of a 3-minute recording in an active state. Participants faced a square on the interface that changed gradually from gray to red, or from gray to blue (i.e., changing saturation), in a random order. Subjects were asked to count the number of saturation changes from gray to red, as a cognitive challenge.

NF Training: Training focused on the enhancement of UA activity over the motor cortex (electrode locations $\mathrm{C} 3$, $\mathrm{Cz}, \mathrm{C} 4, \mathrm{CP} 3, \mathrm{CPz}$ and $\mathrm{CP} 4$, which are referred to as training locations). UA was defined using the Individual Alpha Frequency (IAF) as an anchor point. IAF was defined per user and training location using the screening activity as the peak frequency in the traditional alpha range of [7.512.5] $\mathrm{Hz}$ [15]. UA was then set to the range [IAF, IAF+2] Hz. Independent Component Analysis (ICA) was computed per user to automatically remove the blinking components. UA power was calculated by means of a sliding window FFT, using a 1 second hamming window with $30 \mathrm{~ms}$ of overlapping. Resolution was set to $0.25 \mathrm{~Hz}$ (1024 points using zero-padding). Averaged UA power across the training locations for the screening was considered as the baseline. Feedback was updated each $30 \mathrm{~ms}$ according to the average UA power for the training locations. The feedback was provided visually as the participants faced a square on a screen, either red or blue according to whether the UA power was higher or lower than the baseline, respectively. The saturation of the square was proportional to the deviation of the UA power from the baseline (saturation scale covered $95 \%$ of the power values). This NF method has been proved to produce significant changes in UA, when compared to a control group administered with sham feedback [13].

\section{Motor Assessment}

A Go/No-Go task was designed based on [14] to measure both SMR desynchronization pattern and motor performance. The assessment was divided into 4 runs of 52 trials each. The trials were structured as in Fig. 1. At a random time instant between 1.5 and 2.5 seconds after the beginning of the trial, a warning 'Go' or 'No-Go' stimulus was displayed. Then, an imperative stimulus appeared with an inter-stimulus delay either $D=0.75 s$ or $D=1.5 s$ after the warning stimulus disappeared. This imperative stimulus was always congruent with the warning one (i.e., if warning stimulus was 'Go', then imperative stimulus was also 'Go', and vice versa). Following the imperative stimulus, '+' symbol stayed until the trial had a duration of 5 seconds. Finally, the screen remained blank for 1.5 seconds as the inter-trial interval. The subjects were instructed to click a mouse button, with their right index finger, immediately after the imperative stimulus when it was a 'Go' trial, and to stay relaxed if it was 'NoGo' trial. The time interval between the 'Go' stimulus and the button pressing was recorded, and provided a measure of reaction time of the subjects. Note that the conditions were balanced: each run included 13 trials of each of the four conditions ('Go' and 'No-Go' by the 2 different interstimulus delays), totaling 52 trials of each condition.

\section{RESUltS}

\section{A. Neurofeedback Trainability}

In order to evaluate the progress of the NF training, the average UA power was computed for all screenings and training trials of each session. Due to the large inter-user variations of UA power, these values were normalized per subject to the UA power in the pre-screening of the first session (Fig. 2). Statistical significance was assessed using paired, one-tailed t-tests.

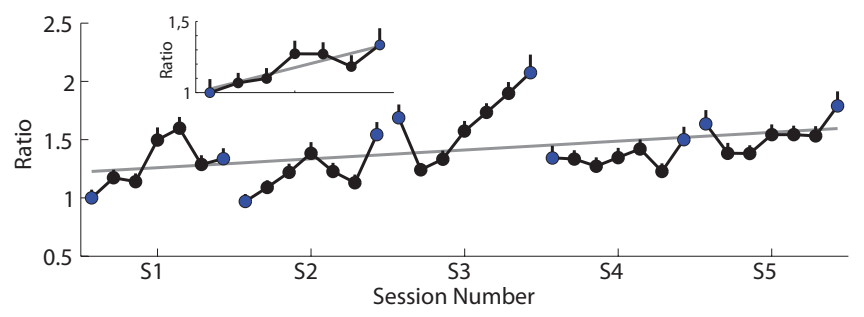

Fig. 2. Average UA power of all participants during the screenings and training trials in each session. In the upper-left corner, the average UA power within sessions is depicted. Blue points denote pre/post screenings, and black points denote training trials. The grey line shows the tendency. Values are normalized for each subject to the UA power in the pre-screening of his/her first session. The vertical bars indicate standard errors of the mean.

Training progress was reflected by a significantly positive tendency of the UA power across the sessions: the gradients 
of a fitted regression line for each subject were significantly larger than zero $(t(4)=2.45, p=0.035)$. Additionally, a positive tendency was also obtained within a session: the gradients of a fitted regression line for each subject (considering the average of sessions) were significantly larger than zero $(t(4)=3.26, p=0.016)$.

\section{B. EEG Analysis}

This section evaluates the changes in EEG patterns during screening and execution of the Go/No-Go tasks. The averaged UA power increased $79 \%$ from the pre-screening of session 1 to the post-screening of session 5. Furthermore, the average UA power within sessions from pre-screening to post-screening was increased 34\%. Regarding IAF values, no significant changes were obtained across subjects and sessions.

For the analysis of the motor task (i.e., Go/No-Go) the entire alpha band was considered for each subject (i.e., $[I A F-2, I A F+2])$ since it contained most information.

The first analysis assessed the time-course representation of alpha power along trials. A variation of the traditional Event-Related Desynchronization (ERD) metric [1] was used. Concretely, the following formula was used to compute the increase ratio with regard to a baseline interval: Rel_Pow $_{t}=$ Pow $_{t} /$ Pow $_{B L}$; where Pow Pow $_{t}$ is the alpha power in time instant $t$; and $P_{0 w_{B L}}$ is the average alpha power of the baseline interval. Note that this metric only assumes a scale change with regard to ERD (i.e., a scale $[-100,100]$ in ERD metric is equivalent to $[0,2]$ scale with the above formula). Baseline was set to the time interval $[-1,0] \mathrm{s}$ (a subset of the rest interval). This metric was separately applied for each condition (Go, No-Go) in pre- and post- assessments. Fig. 3a-b shows the results averaged for all subjects of a $\mathrm{Go} / \mathrm{No}-\mathrm{Go}$ condition (ones with $D=1.5 \mathrm{~s}$ ). Desynchronization can be only observed for the Go condition approximately at $t=2.5 \mathrm{~s}$, which was approximately $50 \%$ (ratio 0.5 ). When comparing preand post- assessment desynchronization values, no statistical differences were found.

However, the aforementioned analysis did not provide information on the effect produced by NF training: the increase of alpha power in rest state. Thus, a second analysis assessed the time-course representation of alpha power, normalizing the post assessment to the baseline interval of the preassessment. Fig. 3c-d show the analogous results of the corresponding figures above them, after normalization. A clear alpha power increase of approximately $50 \%$ (ratio 1.5) in the rest interval can be observed in both Go and No-Go conditions. This increase is not found while clicking with the mouse. Thus, it can be concluded that NF training did not produce observable changes with the variation of the ERD metric, but it produced an increase in desynchronization measured in absolute terms.

\section{BCI Features}

Given that the NF training produced an increase in the desynchronization metric, if a classification problem between
Rest and Task was considered, the separability of the Rest and Task classes would be higher after NF.

In order to assess the statistical significance of the results, a subset of the rest and task intervals was considered. Two time windows of 0.25 s were considered to isolate the desynchronization phenomenon from the subsequent synchronization. The time-window of the Rest class started $0.75 \mathrm{~s}$ before the warning stimulus, while the time-window of the Task class started $0.75 \mathrm{~s}$ after the imperative stimulus was shown. Windows are displayed in Fig. 3c-d with green and magenta colors, respectively. The power distributions (mean and $95 \%$ confidence intervals) are shown in Fig. 4a-b for each of the combinations ([Pre- Post-] by [Rest Task]), averaged for all subjects. Confidence intervals were computed using a t-percentile bootstrap method. Let $\delta 1$ be the distance between means of the power distributions in Rest and Task classes. $\delta 1$ provides information about the distance between distributions-centers. $\delta 2$ measures the distance between the upper confidence interval of the Task distribution (only for Go condition) and the mean Rest distribution. Note that $\delta 2$ actually provides a value of how separable the distributions are (with $95 \%$ significance).

Fig. 4a shows that, for Go trials, the separability between distribution means was significantly increased after NF training (i.e., $\delta 1^{\prime}>\delta 1 ; t(9)=2.36, p=0.02$ ). Additionally, separability between classes was also significantly increased (i.e., $\delta 2^{\prime}>\delta 2 ; t(9)=2.11, p=0.032$ ). On the other hand, Fig. $4 \mathrm{~b}$ shows that, for No-Go trials, there was an increase in the separability between distribution means, but this increase was not significant. Metric $\delta 2$ could not be computed in NoGo trials since upper confidence interval of Task distribution was not lower than the mean of Rest distribution.

\section{Behavioral Analysis}

The mean reaction time for all subjects in the execution of the motor assessment was $335.18 \pm 54.82 \mathrm{~ms}$ before the NF training, and $315.62 \pm 33.47 \mathrm{~ms}$ after NF training. Thus, subjects performed better after NF training, but the time decrease was not significant $(t(4)=1.51, p=0.1)$. Analysis of error occurrences did not show significant differences, which could be due to the simplicity of the task.

\section{CONCLusions}

This paper shows how neurofeedback (NF) training increases the differentiation of motor patterns in healthy users during a motor task execution. With NF training, UA activity was significantly increased for all subjects across training sessions. This increase led to an increase in the SMR desynchronization during the execution of a motor assessment after NF application. Desynchronization was found when measuring absolute power values, which in turn shows an increase in the separability between rest and task intervals in alpha band power. Note that this result may yield better classification performances in BCIs that rely on the usage of EEG activity of the motor cortex. Additionally, performance measurements in the reaction time task were also improved, although this improvement was not significant. 


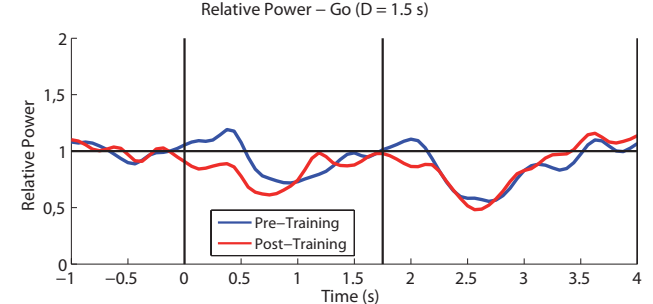

(a)

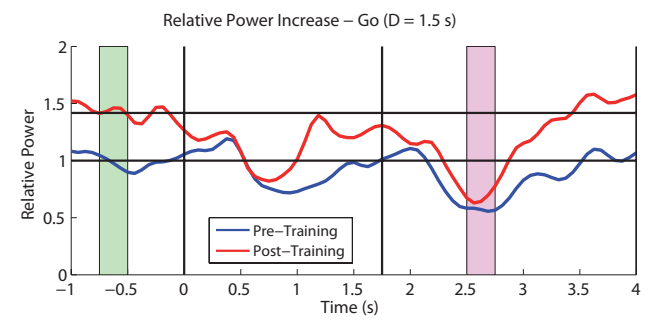

(c)

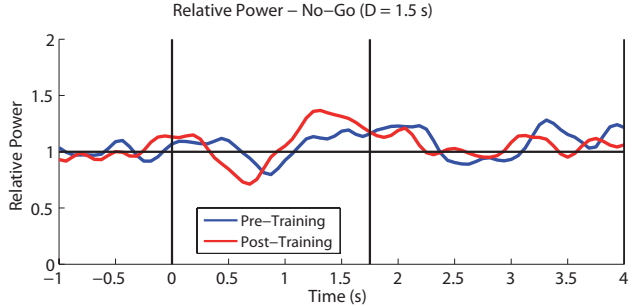

(b)

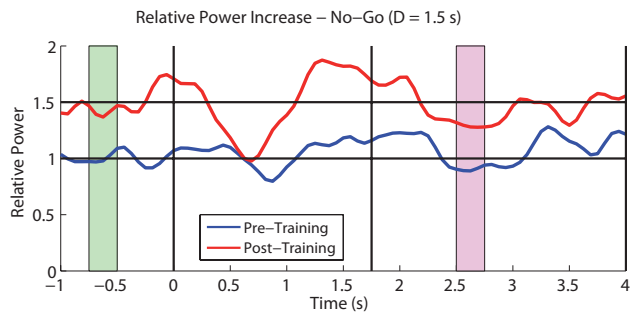

(d)

Fig. 3. Alpha power time course averaged for all subjects. Left and right column show the Go and No-Go trials, respectively, which belong to the inter-stimulus condition $D=1.5 \mathrm{~s}$. (a-b) Relative power of pre- and post- assessments, normalized to the their own baseline interval. (c-d) Relative power of pre- and post- assessments, normalized to the baseline interval of pre- assessment. Green and magenta selections indicate representative time intervals of Rest and Task intervals, respectively.

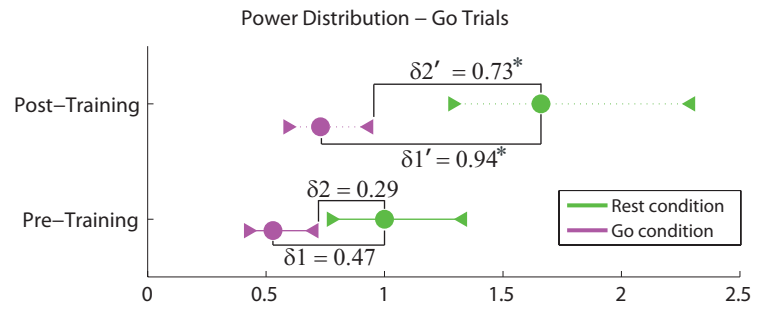

(a)

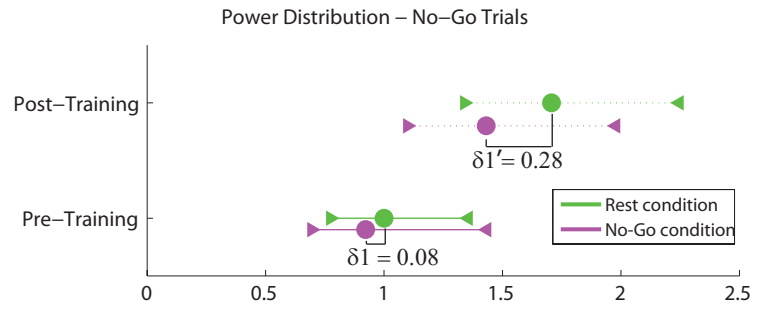

(b)

Fig. 4. Distributions (mean plus 95\% confidence intervals) of the average power of time intervals selected in Fig. 3(c)-(d). (a) Power distributions in preand post- assessments for Go trials. (b) Power distributions in pre- and post- assessments for No-Go trials. Values of metrics $\delta 1$ and $\delta 2$ are presented for each case. The statistically significant values are marked with an asterisk.

\section{REFERENCES}

[1] G. Pfurtscheller and F.H. Lopes da Silva, "Event-related EEG/MEG synchronization and desynchronization: basic principles," Clinical Neurophysiology, vol. 110, no. 11, pp. 1842 - 1857, 1999.

[2] E. López-Larraz, J.M. Antelis, L. Montesano, A. Gil-Agudo, and J. Minguez, "Continuous decoding of Motor Attempt and Motor Imagery from EEG Activity in Spinal Cord Injury Patients," in 34th Annual International Conference of the IEEE Engineering in Medicine and Biology Society (EMBS), 2012.

[3] J.dR. Millán, R. Rupp, G.R. Müller-Putz, R. Murray-Smith, C. Giugliemma, M. Tangermann, C. Vidaurre, et al., "Combining brain-computer interfaces and assistive technologies: state-of-the-art and challenges," Frontiers in neuroscience, vol. 4, 2010.

[4] B. Blankertz, C. Sannelli, S. Halder, E.M. Hammer, A. Kübler, K-R. Müller, G. Curio, and T. Dickhaus, "Neurophysiological predictor of SMR-based BCI performance," Neurolmage, vol. 51, no. 4, 2010.

[5] G.R. Müller-Putz, D. Zimmermann, B. Graimann, K. Nestinger, G. Korisek, and G. Pfurtscheller, "Event-related beta EEG-changes during passive and attempted foot movements in paraplegic patients," Brain Research, vol. 1137, pp. 84 - 91, 2007.

[6] M. Stepien, J. Conradi, G. Waterstraat, F.U. Hohlefeld, G. Curio, and V.V. Nikulin, "Event-related desynchronization of sensorimotor EEG rhythms in hemiparetic patients with acute stroke," Neuroscience Letters, vol. 488, no. 1, pp. 17-21, 2011.

[7] J.M. Antelis, L. Montesano, A. Ramos-Murguialday, N. Birbaumer, and J. Minguez, "Continuous decoding of intention to move from contralesional hemisphere brain oscillations in severely affected chronic stroke patients," in 34th Annual International Conference of the IEEE Engineering in Medicine and Biology Society (EMBS), 2012.
[8] S. Cramer, E. Orr, M. Cohen, and M. Lacourse, "Effects of motor imagery training after chronic, complete spinal cord injury," Experimental Brain Research, vol. 177, pp. 233-242, 2007.

[9] C. Escolano, M. Aguilar, and J. Minguez, "EEG-based upper alpha neurofeedback training improves working memory performance," in 33rd Annual International Conference of the IEEE Engineering in Medicine and Biology Society (EMBS), Boston (USA), 2011.

[10] S. Hanslmayr, P. Sauseng, M. Doppelmayr, M. Schabus, and W. Klimesch, "Increasing individual upper alpha power by neurofeedback improves cognitive performance in human subjects," Applied Psychophysiology and Biofeedback, vol. 30, pp. 1-10, 2005.

[11] C. Neuper, A. Schlögl, and G. Pfurtscheller, "Enhancement of leftright sensorimotor EEG differences during feedback-regulated motor imagery," Journal of Clinical Neurophysiology, vol. 16, no. 4, pp. 373-382, July 1999.

[12] H.J. Hwang, K. Kwon, and C.H. Im, "Neurofeedback-based motor imagery training for braincomputer interface (bci)," Journal of Neuroscience Methods, vol. 179, no. 1, pp. 150 - 156, 2009.

[13] C. Escolano, B Olivan, Y. Lopez-del Hoyo, J. Garcia-Campayo, and J. Minguez, "Double-Blind Single-Session Neurofeedback Training in Upper-Alpha for Cognitive Enhancement of Healthy Subjects," in 34th Annual International Conference of the IEEE Engineering in Medicine and Biology Society (EMBS), 2012.

[14] C.B. Boulay, W.A. Sarnacki, J.R. Wolpaw, and D.J. McFarland, "Trained modulation of sensorimotor rhythms can affect reaction time," Clinical Neurophysiology, vol. 122, no. 9, pp. 1820-1826, 2011.

[15] W. Klimesch, "EEG alpha and theta oscillations reflect cognitive and memory performance: a review and analysis," Brain Research Reviews, vol. 29, no. 23, pp. 169 - 195, 1999. 